\title{
Using socioeconomics to counter health disparities arising from the covid-19 pandemic
}

Principles and methods drawn from decades of work showing that lower socioeconomic status is associated with poorer health should guide efforts to monitor and mitigate the impact of the covid-19 pandemic argue Geoffrey Anderson and colleagues

\section{Geoffrey Anderson professor ${ }^{1}$, John William Frank professor ${ }^{2}$, C David Naylor professor ${ }^{3}$, Walter Wodchis professor ${ }^{1}$, Patrick Feng adjunct professor ${ }^{4}$}

'Institute of Health Policy Management and Evaluation, University of Toronto, Toronto, Ontario, Canada; ${ }^{2}$ Usher Institute, University of Edinburgh, Edinburgh, UK; ${ }^{3}$ Department of Medicine, University of Toronto, Toronto, Canada; ${ }^{4}$ Strategic Foresight and Innovation Program, OCAD University, Toronto, Canada

Epidemiological models have predicted that without interventions to contain the spread of covid-19, countries would face an exponential increase in cases. ${ }^{1}$ Although most of those cases will be mild, a meaningful minority of people would fall seriously ill, potentially overwhelming hospitals and resulting in a sharp increase in deaths. These scenarios have led many countries to adopt measures aimed at "flattening the curve" to avoid a sudden spike in covid-19 cases. The strategies are predominantly based on reducing close contact between individuals to lower the chances of transmission. However, social distancing strategies could have profound effects on health through various mechanisms, including employment, social isolation, and effects on family relationships. ${ }^{2}$ Furthermore, concern is growing that poor and vulnerable people will bear the brunt of both the virus and strategies to contain it. ${ }^{23} \mathrm{We}$ urgently need to measure and mitigate differential effects of the pandemic on already marginalised populations.

We recommend that assessments of the covid-19 pandemic and measures to contain it be informed by well established principles and methods that consider the complex interplay between socioeconomic status and health disparities. Furthermore, we argue these principles can provide a framework ${ }^{4}$ to guide strategies to ease physical distancing measures and equitable policies to deal with the pandemic's long term effects on health and society.

\section{Socioeconomic status and health gradient}

Years of research from many high income countries has shown that health is related to socioeconomic status in important and complex ways. The underlying principles for that research build on work done decades ago, mostly in England, that argued inequalities in health related to socioeconomic status are a consequence of inequalities in the social determinants of health.
These social determinants include material circumstances, the social environment, and psychological factors. These are in turn influenced by social position and context and shaped by a range of factors, including education, income, and ethnicity. Furthermore, these health inequities exist not only between the extremes of rich and poor but across every rung on the socioeconomic status ladder.

Marmot argues these differences are not primarily driven by income but have more to do with variations in social participation and ability to control life circumstances. ${ }^{6}$ This mechanism has been highlighted in more recent work by Case and Deaton in the United States, who argue that loss of employment certainty and social opportunity are associated with sharp increases in deaths tied to despair, including suicides, and deaths related to alcohol and drug dependency in middle class, middle aged, non-Hispanic white people. ${ }^{7}$ Recent work in Canada has used constructs of socioeconomic risks such as food insecurity and housing instability to show how these can predict future high use of healthcare services. ${ }^{8}$ Scotland has moved to regular reporting of socioeconomic related health disparities using a sophisticated multifaceted deprivation index. ${ }^{9}$ Along with research and reporting within countries, studies have looked at socioeconomic related health disparities across countries. ${ }^{1011}$

\section{Monitoring the effects of covid-19 policies}

These studies highlight two key conceptual themes: firstly, socioeconomic status is multifaceted and should be measured as far more than just income; and, secondly, socioeconomic characteristics are consistently related to a range of outcomes including disease incidence, mortality, and healthcare use. Empirically, this relation often occurs as a gradient across socioeconomic groups and this socioeconomic status gradient 
has both individual ${ }^{6-8}$ and community level ${ }^{9-11}$ associations with health outcomes.

It is not difficult to imagine that the effects of material and social deprivation that disadvantage poor people generally (eg, lack of resources and social isolation) are also at play in the covid-19 pandemic. Marginalised groups face special risks. They may be more likely to become infected because of cramped living conditions and the relative lack of resources to self-isolate and physically distance. They also have higher rates of many of the comorbidities such as hypertension and diabetes that predict poor outcomes for those who are infected. Those monitoring outcomes of the pandemic and response should take into account both individual socioeconomic status and the social determinants of the communities in which individuals live.

This type of analysis is starting to appear, including a recent report from Canada that showed people living in marginalised neighbourhoods-as measured by ethnic concentration, residential instability, material deprivation, and income-are more likely to test positive for covid-19 and that each of these measures have different effects. ${ }^{12}$ Ecological analyses such as these, using postcodes linked to small area data from census data or social surveys, are a powerful and efficient approach to socioeconomic status based analyses.

A key attribute of sound ecological analysis is creating local areas that reflect neighbourhoods in a true community sense. For example, Scotland uses 7000 areas that have been carefully crafted to capture neighbourhoods ranging from public housing estates to wealthy enclaves to cover a population of 5.45 million. ${ }^{9}$ Analysis based on individual level socioeconomic status data on covid-19 complications is also starting to appear. For example, a recent analysis by the UK Office for National Statistics of covid-19 death rates shows nearly fourfold higher mortality in unskilled and manual workers compared with professionals. ${ }^{13}$ These early analyses are showing the important and varied nature of socioeconomic status disparities for this new threat to health.

A recent $B M J$ article on the effects of covid $-19^{2}$ containment measures suggests a socioeconomic status lens can identify important effects of pandemic responses, including responses of healthcare systems. The large and sudden effects of job loss and concerns over future employment combined with drastic changes in social and family context ${ }^{2}$ raise the possibility of an increase in Case and Deaton's deaths of despair. ${ }^{7}$ These deaths were primarily but not uniquely observed in the US and are already being talked about as a potential epidemic within the pandemic in that country. ${ }^{14}$

Violence against others, such as domestic violence and child abuse, may also be important markers of the socioeconomic related effects of covid-19 containment. ${ }^{2}$ Longer term material and social deprivation, combined with restricted access to health services in an increasingly hard to access and strained health and social care system, could affect healthcare use and health outcomes in populations with complex health and social care needs, such as people who are old, frail, or have multiple chronic conditions or serious mental illness and addictions. ${ }^{15}$

In short, proper monitoring of covid-19 should examine both the direct effects of covid-19 and the health effects of containment policies using comprehensive measures of material and social deprivation at both individual and ecological level. Multidimensional socioeconomic deprivation indices, derived from census data and population based surveys, should be used to support ecological analyses. These indices are most useful when mapped to geographical areas whose boundaries respect natural communities that are relatively homogeneous in terms of socioeconomic status. Postcode information routinely collected from healthcare encounters or vital statistics is typically used to map events in individuals to these geographical areas, with census data providing the denominator for calculating rates in terms of the components of the deprivation index for that geographical area.

A related priority should be to look carefully at the data routinely collected at individual level for important outcomes that can be used to discern socioeconomic disparities. Those planning the response should be willing to invest in new forms of individual level data on socioeconomic status that can guide efforts to protect and support those at risk or those who have been disproportionately burdened.

\section{Guide for exit strategies and social recovery}

Leaders in socioeconomic health research have made a point of describing the policy implications of their work..$^{5-7}$ Whitehead defined four categories that link the theory and measurement of socioeconomic status health inequities to different levels of policy: strengthening individuals, strengthening communities, improving living and working conditions, and macro-policies addressing the broader determinants of health. ${ }^{4}$ This classification should be used to inform strategies for exiting lockdown measures and guide investments to support social and economic recovery (box 1).

Box 1: Using health-wealth gradients to guide strategies for covid-19 containment and social recovery

Policies aimed at individuals such as immunity passports should take into account the disparities in health benefits of being able to return to society across socioeconomic groups and be implemented with careful attention to both equitable access to testing and measures required to mitigate the socioeconomic differences in financial and social advantages accruing to those who are shown to be immune

Policies aimed at protecting communities through specific isolation strategies and contact tracing should be designed to protect the most vulnerable, such as residents of long term care facilities, homeless people, and marginalised ethnic groups

Policies on working conditions should mitigate differences in risk of infection by employment sector, and ensure those whose employment is central to economic recovery are provided with adequate protection and support in the workplace

Macro-policies aimed at broad mitigation of economic effects should include programmes that provide targeted support to those most affected and those who face greater obstacles in re-entering society (eg, people who are precariously employed, homeless, or have complex needs)

At the individual level, the concept of "immunity passports" has received considerable attention. Experts caution that the extent and duration of protection conferred by antibodies to SARS-CoV-2 is still unclear, but technical issues are far from the only concern about this idea. If antibodies are found to confer durable immunity, these tests could have immediate implications for who can (or cannot) resume in-person activities, generating a whole host of equity issues. Antibody tests could become a new gatekeeper to employment in congregate workplaces (typically paid hourly at lower levels) while salaried executives and professionals continue to work remotely regardless of immunity status. Another issue is whether antibody tests will be sold privately or offered as a publicly insured service. Policies on this front must be framed with careful attention to the potential exacerbation of existing socioeconomic disparities.

At the community level, there have been extensive discussions around options to develop capacity for testing and contact tracing on a scale seen in countries such as South Korea, Hong Kong, and Singapore. Sustaining this capacity is particularly important to control spread as physical distancing restrictions 
are eased. A socioeconomic status disparities lens would lead us to focus on protecting the most vulnerable members of our society, such as residents of nursing homes and long term care facilities, ${ }^{16}$ homeless people, ${ }^{17}$ and marginalised ethnic groups; it would also pay attention to systemic factors such as historical and ongoing racism. ${ }^{18}$ These groups seem to have slipped through the cracks in the initial response in many countries, and they merit special attention as the first wave of the covid-19 epidemic recedes and subsequent waves threaten.

Technology driven solutions such as digital contact tracing have been proposed in the context of both individual and community level policy responses. These digitals tools raise equity concerns (eg, access, privacy, digital divide) across socioeconomic strata that must be taken into account as these are developed and implemented. ${ }^{19}$ Likewise, broad serosurveillance studies-important in shaping outbreak management and vaccination policy-must be designed so that marginalised populations are not excluded.

Working conditions are another area of great interest. In Canada, employees of food processing plants were deemed essential workers. Meat packing plants, in particular, involve unattractive work at close quarters, pay low wages, and often are staffed by immigrants and people belonging to ethno-racial minorities. Major outbreaks of covid-19 have occurred in these facilities in both Canada and the US. Similar workplace outbreaks are likely to be seen globally as economic restrictions are lifted. As trades unions in the UK have warned, ${ }^{20}$ guidelines for employers to keep employees safe are vague and monitoring procedures unclear. Clearly, congregate settings with common work areas such as factories create huge risks for workers; by contrast, white collar employers can erect partitions in offices, stagger hours, limit meeting sizes, and more readily maintain some of the working from home arrangements used during containment.

At a macro-level, many high income countries are introducing economic measures to offset financial difficulties faced by citizens because of the pandemic. Tactics vary, including subsidies to employers to prevent redundancies and direct payments to families to mitigate hardship. It is not clear, however, that these policies have been tailored to ensure support for those who are most precariously employed, or those with limited means or compromised immune systems. More generally, as jurisdictions begin to ease containment measures and restore the functions of civil society, healthcare systems, and economic activities, it is easy to overlook the complex interactions between socioeconomic status and health. These interactions warrant special attention in the challenging months ahead.

\section{Rebuilding fairly}

The epidemic curve has provided an important framework for understanding and containing the spread of covid-19. We believe the socioeconomic-health disparities gradient provides an equally important framework-one that can deepen understanding of the differing health effects of covid-19 and containment strategies across socioeconomic groups. Governments and population health researchers should collect detailed and meaningful data on the socioeconomic distribution of both the direct health effects of the pandemic and the indirect health, social, and economic effects resulting from covid-19 containment strategies. The socioeconomic lens can also provide policy makers with useful guidance as they develop and deploy strategies to exit containment and make investments to mitigate, in an equitable way, the longer term effects of this pandemic.
Covid-19 has made the world less healthy. Responses to it need not make the world less equitable. With careful attention to principles, methods, and policy ideas that come from over two decades of research and ideas, countries can better anticipate, mitigate, and redress the health and social effects of this pandemic-particularly on the most marginalised groups in society.

\section{Key messages}

Early data suggest both the incidence and effect of covid-19 will be distributed unequally across those with different levels of material and social deprivation

Strategies to contain covid-19 are greatly affecting key social determinants of health such as employment, social interaction, and family relationships

People with complex needs, vulnerable populations, and marginalised groups are at increased risk from covid-19 and the health effects of containment strategies

Timely, reliable data are needed to identify these individuals and ensure they are properly supported

The socioeconomic disparities in health gradient provide an important framework to deepen understanding of, and mitigate, the health equity effects of covid-19

Contributors and sources: The authors worked together on the initial manuscript and revisions and all shared in the design, literature review, and drafting of the manuscript. GA has been involved in research on health system performance, socioeconomic determinants of health, and access and international comparative analysis for three decades. JWF is an academic public health physician who has focused his work over the past three decades on the broader determinants of health and has been in academic leadership positions in public health in Canada, the US, and most recently Scotland. CDN is a former president of the University of Toronto and chaired the Canadian National Advisory Committee on SARS and public health. WW has conducted Canadian and international studies on socioeconomic status and health and healthcare use and led international comparisons of care for high needs population.PF is a Fulbright scholar whose research spans health and innovation policy. GA is the guarantor of the article.

Competing interests: We have read and understood BMJ policy on declaration of interests and have no relevant interests to declare.

Provenance and peer review: Not commissioned; externally peer reviewed.

1 Anderson RM, Heesterbeek H, Klinkenberg D, Hollingsworth TD. How will country-based mitigation measures influence the course of the COVID-19 epidemic? Lancet 2020;395:931-4. 10.1016/S0140-6736(20)30567-5 32164834

2 Douglas M, Katikireddi SV, Taulbut M, McKee M, McCartney G. Mitigating the wider health effects of covid-19 pandemic response. BMJ 2020;369:m1557. 10.1136/bmj.m1557 32341002

3 What COVID-19 is teaching us about inequality and the sustainability of our health systems. EuroHealthNet, 5 May 2020. https://eurohealthnet.eu/COVID-19

4 Whitehead M. A typology of actions to tackle social inequalities in health. J Epidemiol Community Health 2007;61:473-8. 10.1136/jech.2005.037242 17496254

5 Marmot M, Goldblatt P, Allen J, et al. Fair society, healthy lives. 2010. http://www. instituteofhealthequity.org/resources-reports/fair-society-healthy-lives-the-marmot-review/ fair-society-healthy-lives-full-report-pdf.pdf

6 Marmot M. The influence of income on health: views of an epidemiologist. Health Aff (Millwood) 2002;21:31-46. 10.1377/hlthaff.21.2.31 11900185

7 Case A, Deaton A. Mortality and morbidity in the 21st century. Brookings Panel on Economic Activity, 23-24 Mar 2017. https://www.brookings.edu/wp-content/uploads/2017/ 03/6_casedeaton.pdf

8 Fitzpatrick T, Rosella LC, Calzavara A, etal . Looking beyond income and education: socioeconomic status gradients among future high-cost users of health care. Am J Prev Med 2015:49:161-71. 10.1016/..amepre.2015.02.018 25960393

9 Frank J, Haw S. Best practice guidelines for monitoring socioeconomic inequalities in health status: lessons from Scotland. Milbank Q 2011;89:658-93. 10.1111/j.1468-0009.2011.00646.x 22188351

10 Cookson R, Mondor L, Asaria M, Kringos DS, Klazinga NS, Wodchis WP. Primary care and health inequality: Difference-in-difference study comparing England and Ontario. PLoS One 2017;12:e0188560. 10.1371/journal.pone.0188560 29182652

11 Billings J, Anderson GM, Newman LS. Recent findings on preventable hospitalizations. Health Aff (Millwood) 1996;15:239-49. 10.1377/hlthaff.15.3.239 8854530

12 Chung H, Fung K, Ferreira-Legere LE, et al. COVID19 laboratory testing in Ontario: patterns of testing and characteristics of individuals tested, as of April 30, 2020. ICES, 2020. https://www.ices.on.ca/Publications/Atlases-and-Reports/2020/COVID-19-LaboratoryTesting-in-Ontario

13 Office for National Statistics. Coronavirus (COVID-19) related deaths by occupation, England and Wales: deaths registered up to and including 20 April 2020. 2020. https:// www.ons.gov.uk/peoplepopulationandcommunity/healthandsocialcare/causesofdeath/ 
bulletins/coronaviruscovid19relateddeathsbyoccupationenglandandwales/ deathsregistereduptoandincluding20april2020

14 Petterson, S, Westfall J, Miller B. Projected deaths of despair during the coronavirus recession. Well Being Trust, 8 May 2020. https://wellbeingtrust.org/wp-content/uploads/ 2020/05/WBT_Deaths-of-Despair_COVID-19-FINAL-FINAL.pdf

15 Bhattacharyya $\mathrm{O}$, Shaw J, Sinha S, etal . innovative integrated health and social care programs in eleven high-income countries. Health Aff (Millwood) 2020;39:689-96. 10.1377/hlthaff.2019.00826 32250690

16 Lloyd-Sherlock PG, Kalache A, McKee M, Derbyshire J, Geffen L, Casas FG. WHO must prioritise the needs of older people in its response to the covid-19 pandemic. BMJ 2020;368:m1164. 10.1136/bmj.m1164 32205399

17 Tsai J, Wilson M. COVID-19: a potential public health problem for homeless populations. Lancet Public Health 2020;5:e186-7. 10.1016/S2468-2667(20)30053-0 32171054

18 Webb Hooper M, Nápoles AM, Pérez-Stable EJ. COVID-19 and racial/ethnic disparities. JAMA 2020;67:166-75. http://doi.wiley.com/10.1002/jclp.20757. 32391864
19 Ada Lovelace Institute. Exit through the App store? A rapid evidence review on the technical considerations and societal implications of using technology to transition from the COVID-19 crisis. 20 Apr 2020. https://www.adalovelaceinstitute.org/our-work/covid19/covid-19-exit-through-the-app-store/

20 Mason R, Stewart H. Anger at UK lockdown easing plans "that could put workers at risk." Guardian 2020 May 4. https://www.theguardian.com/uk-news/2020/may/04/uk-unionscriticise-guidance-on-returning-to-work-for-being-inadequate

This article is made freely available for use in accordance with BMJ's website terms and conditions for the duration of the covid-19 pandemic or until otherwise determined by BMJ. You may use, download and print the article for any lawful, non-commercial purpose (including text and data mining) provided that all copyright notices and trade marks are retained.

https://bmj.com/coronavirus/usage 